regard to the retrospective study design, the findings challenge current guideline recommendations regarding PHECG in patients with acute stroke.

Conflict of interest None

Funding University of Surrey School of Health Sciences PhD scholarship.

\section{PARENTS' EXPERIENCES OF THE CARING ENCOUNTER IN THE AMBULANCE SERVICE- A QUALITATIVE INTERVIEW STUDY}

1,2,3 K Karlsson* 2,3,4 K Rooth 1,2,3,4V Lindström. ${ }^{1}$ Samariten Ambulance Stockholm Sweden; ${ }^{2}$ Academic EMS, Stockholm, Sweden; ${ }^{3}$ Karolinska Institutet, Department of Neurobiology, Care Sciences, and Society Division of Nursing Stockholm, Sweden; ${ }^{4}$ The Ambulance Medical Service in Stockholm (AISAB), Sweden

\subsection{6/10.1136/bmjopen-2018-EMS.15}

Aim Care provided by the ambulance service is often associated with emergency medicine, traumatology and disaster medicine this leaving a rather scant volume of research on children and their parents' perspective on the care received. Aim: To explore parents' experience of caring encounter in the ambulance service.

Method A qualitative design with interviews and an inductive qualitative content analysis was used.

Results 14 parents described 16 encounters' with ambulance personnel. Overall they expressed satisfaction with the care their children received. The parents described the significance when the personnel gave the family enough time, created a safe environment and involved the parents in the care. The parents felt unsafe when there were lack of communication and sensitivity, the equipment was not adaptable for children, and the parents was not invited to participate in the care.

Conclusion In order to improve the care in the ambulance it is imperative that we understand what parents want and value. Overall, parents' experiences was positive, but there is a need to strengthen the family-centred care in the ambulance service. Not inviting the parents to participate in the care, using not adaptable equipment caused a sense of uncertainty, lack of trust and increased level of stress among the parents. This may be caused by a lack of training or knowledge on caring of children among the personnel, managers need to acknowledge this in the ambulance personnel's continues medical education. Further intervention studies aiming to develop the family-centred care in the ambulance service is needed.

Conflict of interest None

Funding Founding was provided by Karolinska Institutet FoBi foundations 2017.

\section{EMERGENCY MEDICAL SERVICES PHYSICIANS' PERCEPTIONS OF AMBULANCE NURSES' RESPONSIBILITY FOR REFERRING PATIENTS TO PRIMARY CARE AND SELF-CARE - A SWEDISH NATIONAL SURVEY}

\footnotetext{
${ }^{1,2,3} \mathrm{~A} \mathrm{Bremer}^{*},{ }^{4} \mathrm{E}$ Kowalczyk, ${ }^{1,5} \mathrm{~K}$ Årestedt, ${ }^{3,6} \mathrm{~B}$ Wireklint Sundström. ${ }^{1}$ Faculty of Health and Life Sciences, Linnaeus University, Växjö/Kalmar, Sweden; ${ }^{2}$ Division of Emergency Medical Services, Kalmar County Hospital, Kalmar, Sweden: ${ }^{3}$ University of Borås, Sweden, PreHospen - Centre for Prehospital Research; ${ }^{4}$ Division of Emergency Medical Services, Helsingborg County Hospital, Helsingborg, Sweden; ${ }^{5}$ The Research Unit, Kalmar County Hospital, Kalmar, Sweden; ' ${ }^{6}$ University of Borås, Sweden, Faculty of Caring Science, Work Life and Social Welfare
}

10.1136/10.1136/bmjopen-2018-EMS.16
Aim Based on guidelines developed by EMS physicians, registered nurses in the Swedish ambulance services sometimes by-pass the emergency department and refer non-urgent patients to primary care and self-care. However, these referrals are associated with problems that may jeopardise patient safety and patient participation. The aim was to identify the EMS physicians' perceptions of ambulance nurses' responsibilities and prerequisites to refer patients to primary care and self-care.

Method A national survey of all EMS physicians $(n=51)$ using study specific questions with close-ended and open response options, analysed with descriptive statistics and thematic analysis.

Results The response rate was $78 \%(n=40)$. The majority of the physicians (95\%) perceived that nurses should be able to refer patients to primary care and self-care. One fourth (25\%) perceived specialist nurses in ambulance care as the most appropriate professionals. The majority of the physicians $(65 \%)$ perceived that referral to primary care maintain patient safety, whereas fewer $(50 \%)$ assessed the referral as safe for the patient. All perceived that feedback should be given to nurses when the referral was incorrect. The majority perceived it important to extend the nurses' authority to refer to primary care (63\%) and self-care (55\%).

Conclusion There is no consensus among the EMS physicians regarding responsibilities and conditions for nurses' referral of patients. Registered nurses with basic education are expected to be able to refer, while there is varying opinion regarding requirements of specialist trained ambulance nurses to refer patients. Professional experience as a nurse is perceived more important than formal education.

Conflict of interest None

Funding Division of Emergency Medical Services, Helsingborg County Hospital, Helsingborg, Sweden.

\section{SUDDEN CARDIAC DEATH: AS SUDDEN AS COMMONLY THOUGHT OR DO WE KNOW OUR PATIENTS BEFORE?}

${ }^{1} \mathrm{HK}$ Heymes*, ${ }^{1,2} \mathrm{SK}$ Beckers, ${ }^{1} \mathrm{~N}$ Lenssen, ${ }^{1} \mathrm{R}$ Rossaint, ${ }^{1,2} \mathrm{M}$ Felzen. ${ }^{1}$ Department of Anaesthesiology, University Hospital RWTH Aachen, Medical faculty RWTH Aachen University, Aachen, Germany; ${ }^{2}$ Medical Direction, Emergency Medical Service, City of Aachen, Aachen, Germany

\subsection{6/10.1136/bmjopen-2018-EMS.17}

Aim About 100.000 people per year suffer from sudden cardiac death in Germany. ${ }^{1}$ In most of the cases $(75 \%)$ this is cardiac-associated. ${ }^{2}$ According to this it appears to be probable that many patients did already have pre-existing cardiac diseases and might have been under medical treatment before. The aim of this study was to evaluate how many patients, who suffered from out-of-hospital cardiac arrest (OHCA), had documented diagnoses in the appropriate CA centre before.

Method In this retrospective, observational study data was analysed based on the local documentation in the German Resuscitation Registry of the Emergency Medical Service of the city of Aachen. This data was matched with documentation of the local CA centre concerning pre-existing conditions and diagnoses for a five-year period (2012 to 2016).

Results Overall 904 patients (Mean age $70 \pm 18$ years) suffered from OHCA. Among these 41,9\% $(n=379)$ have been under medical treatment in the university hospital of Aachen during the last $3,7 \pm 4,5$ years. $50,9 \% \quad(n=193)$ of these had cardiopulmonary issues and $42,5 \%(n=161)$ were under medical treatment because of cardiac problems.

Conclusion The analysis shows that more than $40 \%$ of these patients, who were resuscitated during the years of 2012 to 